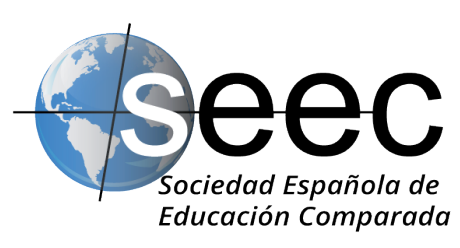

\title{
¿Quién le pone el cascabel al gato? Un análisis comparativo de las agencias de garantía de la calidad de la educación superior
}

Who is going to bell the cat? A comparative analysis of higher education quality assurance agencies

\section{Francesc Pedró*}

DoI: $10.5944 /$ reec.37.2021.27880

Recibido: 9 de julio de 2020 Aceptado: 14 de diciembre de 2020

\footnotetext{
Francesc Pedró: Actualmente es Director del Instituto Internacional de la UNESCO para la Educación Superior en América Latina y el Caribe (UNESCO IESALC). Anteriormente, ha liderado el servicio de asesoramiento en políticas educativas de la UNESCO. Trabajó también en el Centro de Investigación e Innovación Educativas (CERI) de la OCDE donde fue responsable de proyectos como Teaching and Learning International Survey (TALIS) y New Millennium Learners Project. Doctor con una tesis sobre educación comparada en la UNED (Madrid). Estudios postdoctorales en Políticas Públicas Comparadas (Instituto de Educación de la Universidad de Londres). Catedrático de políticas comparadas de educación (Universitat Pompeu Fabra, Barcelona). Ha sido Vicerrector de investigación educativa e innovación de la Universitat Oberta de Catalunya (Barcelona). Datos de contacto: E-mail: f.pedro@unesco.org
} 


\title{
Resumen
}

Las agencias de aseguramiento de la calidad de la educación superior se han expandido por todo el mundo en muy poco tiempo como un intento de profesionalizar la regulación pública de unos procesos de enorme complejidad de un sector caracterizado por una proverbial autonomía de las instituciones. Esta contribución presenta un análisis comparativo de las razones que han llevado a semejante expansión, así como de las convergencias y las divergencias que emergen globalmente. A pesar de las diferencias contextuales, en las que las distintas configuraciones de agencias operan, lo cierto es que existen unas problemáticas comunes que son prácticamente universales, entre las que se cuentan la cuestión de su independencia y su relativa incapacidad de rendir cuentas. La contribución arroja luz, finalmente, sobre las principales críticas que reciben y que tienen que ver, fundamentalmente, con el riesgo de la captura normativa por parte de las propias instituciones de educación superior a las que, en principio, debería regular.

Palabras clave: educación superior; aseguramiento de la calidad; agencias.

\begin{abstract}
Higher education quality assurance agencies have expanded around the world in a very short time as an attempt to professionalize the public regulation of processes of enormous complexity in a sector characterized by a proverbial autonomy of institutions. This contribution presents a comparative analysis of the reasons that have led to such an expansion, as well as the convergences and divergences that are emerging worldwide. Despite the contextual differences, in which the various configurations of agencies operate, the truth is that there are common problems that are practically universal, including the question of their independence and their relative inability to externally be accountable. Finally, the contribution sheds light on the main criticisms they receive, which relate primarily to the risk of regulatory capture by the higher education institutions themselves, which they are supposed to regulate.
\end{abstract}

Keywords: higher education; quality assurance; agencies. 


\section{Introducción}

El establecimiento por parte de los gobiernos de agencias especializadas para la gestión de áreas de interés público de elevada complejidad técnica ha crecido en los últimos años. Gran parte de la aplicación, el control y la reglamentación de las políticas públicas se ha transferido a organismos autónomos, separados de la administración central. Esas organizaciones especializadas, con un solo propósito, han desmenuzado la administración básica tradicional en partes más pequeñas, tanto vertical como horizontalmente. Si bien la tendencia es bastante generalizada, las agencias distan mucho de ser homogéneas. El hecho de que las agencias tomen una gran variedad de formas y tamaños está bien documentado (Pollitt, Bathgate, Caulfield, Smullen, \& Talbot, 2001). Talbot (2004) define a las agencias de manera bastante restrictiva, como un organismo que se separa formalmente de un ministerio, realiza tareas públicas de forma permanente, se financia principalmente con el presupuesto estatal, cuenta con funcionarios públicos y está sujeta a procedimientos legales públicos.

Las agencias de garantía de calidad de la educación superior (AGC, en adelante) son, por su propia naturaleza, una instancia sectorial de organismos reguladores. Las AGC deben garantizar la credibilidad de los procesos de acreditación y revisión de programas e instituciones y garantizar la objetividad y la transparencia de sus decisiones o recomendaciones (Martin \& Stella, 2007). Las AGC desempeñan un papel importante en la dinámica cambiante entre el Estado y las universidades. En la mayoría de los países, existe una relación de tensión entre las universidades, que pregonan su proverbial autonomía, y el Estado, que no solo financia las públicas, sino que quiere garantizar que la educación superior sirva a los intereses nacionales. En la tensión entre autonomía y control, las AGC desempeñan una importante función de mediación en esta relación.

En efecto, las agencias son un factor definitorio del denominado «estado regulador», un estado donde el gobierno no presta servicios pero delega las tareas a entidades privadas o entidades públicas autónomas y utiliza la regulación por medio de las agencias interpuestas para dirigir la orientación y calidad de la prestación (Majone, 2008). La regulación tiene lugar en el triángulo entre demanda política (parlamento, gobierno), regulador (agencias), y regulado (universidades). En esta relación, sin embargo, los organismos reguladores independientes tienden a obtener un considerable poder de formulación de políticas y se convierten en actores con sus propios intereses hasta el punto de que pueden generar dificultades en lo que debiera ser un proceso de alineación con las prioridades nacionales. Como sucede en muchos hogares, los gobiernos tienen ahora un gato en casa, con las ventajas y los inconvenientes que este conlleva. $\mathrm{Y}$, como reza el dicho, quizás el más importante sea que es extremadamente difícil colocarle un cascabel para así poder saber en cada momento dónde está y, al mismo tiempo, evitar que se escape. Difícil tarea, pero no imposible.

\section{Antecedentes, objetivos y metodología}

La investigación comparativa no ha sido, hasta el momento, muy pródiga en el análisis de las tendencias internacionales sobre la configuración y evolución de las AGC (Juanatey, Jordana, Durán, \& Royo, 2020). Abundan las descripciones, particularmente a escala regional para América Latina (Fernández Lamarra, 2006; Strah, 2020), Asia (Yung-Chi Hou, Ince, Tsai, \& Chiang, 2015) o Europa (Alzafari \& Ursin, 2019), pero la investigación 
orientada a proveer evidencias para informar las decisiones políticas, tanto a escala gubernamental (UNESCO IESALC, 2020) como del sector o institucional (Seyfried \& Pohlenz, 2018), sigue siendo escasa.

En este contexto, esta contribución tiene un doble objetivo: en primer lugar, ofrecer un marco analítico para mejorar la comprensión de las AGC desde una perspectiva política y, en segundo lugar, presentar un análisis comparativo de una muestra de ellas. La hipótesis subyacente es que se está produciendo una gran convergencia a escala internacional que no es otra cosa que la expresión de la creciente globalización y homogeneización de la educación superior. De ahí que los retos a los que se enfrentan sean compartidos.

Metodológicamente, el análisis se fundamenta en una revisión de la literatura y el análisis de diez casos que fueron seleccionados por ser los que más atención internacional han recibido. Para seleccionarlos, se tomaron las diez que aparecen más citadas en SCOPUS y que resultaron ser las de los siguientes países: Argentina, Australia, Canadá, Chile, Colombia, Corea del Sur, España, Estados Unidos, México. La Tabla 1 ofrece los detalles de cada una de ellas. El estudio de cada caso se basó en una revisión de la literatura y, en segundo lugar, de los correspondientes sitios web y las publicaciones institucionales incluidas en ellos.

Tabla 1.

Agencias seleccionadas, por países y regiones.

\begin{tabular}{|c|c|c|c|}
\hline Región & País & Agencia & Nombre completo \\
\hline \multirow[t]{2}{*}{ Asia y el Pacífico } & Australia & TEQSA & $\begin{array}{l}\text { Tertiary Educational } \\
\text { Quality and Standards } \\
\text { Agency }\end{array}$ \\
\hline & Corea del Sur & KUAI & $\begin{array}{l}\text { Korean University } \\
\text { Accreditation } \\
\text { Institution }\end{array}$ \\
\hline \multirow[t]{2}{*}{ Europa } & España & ANECA & $\begin{array}{l}\text { Agencia Nacional } \\
\text { de Evaluación de la } \\
\text { Calidad y Acreditación }\end{array}$ \\
\hline & Reino Unido & AGC & $\begin{array}{l}\text { Quality Assurance } \\
\text { Agency }\end{array}$ \\
\hline \multirow[t]{4}{*}{ América Latina } & México & COPAES & $\begin{array}{l}\text { Consejo para la } \\
\text { Acreditación de la ES }\end{array}$ \\
\hline & Argentina & CONEAU & $\begin{array}{l}\text { Comisión Nacional } \\
\text { de Evaluación } \\
\text { y Acreditación } \\
\text { Universitaria }\end{array}$ \\
\hline & Colombia & $\mathrm{CNA}$ & $\begin{array}{l}\text { Consejo Nacional de } \\
\text { Acreditación }\end{array}$ \\
\hline & Chile & $\mathrm{CNA}$ & $\begin{array}{l}\text { Comisión Nacional de } \\
\text { Acreditación }\end{array}$ \\
\hline \multirow[t]{2}{*}{ América del Norte } & Estados Unidos & CHEA & $\begin{array}{l}\text { Council for } \\
\text { Higher Education } \\
\text { Accreditation }\end{array}$ \\
\hline & $\begin{array}{l}\text { Canada, Provincia de } \\
\text { Ontario }\end{array}$ & OUCQA & $\begin{array}{l}\text { Ontario Universities } \\
\text { Council on Quality } \\
\text { Assurance }\end{array}$ \\
\hline
\end{tabular}

Fuente: Elaboración propia. 
Las páginas que siguen presentan, en primer lugar, el marco analítico que permite entender el origen de las AGC y su evolución más reciente a escala global. Sin embargo, debido a los distintos contextos históricos y políticos, el análisis comparativo demuestra que existen distintos modelos, que son presentados en segundo lugar, y que demuestran alternativas de gobernanza. En tercera lugar, se presentan los métodos más comúnmente utilizados como un indicador claro de la creciente convergencia internacional a pesar de la aparente diversidad de modelos. En cuarto lugar, se sugieren cuáles son las dificultades con las que estos modelos tropiezan y que, de nuevo, resultan ser convergentes: la independencia, la rendición de cuentas y la necesidad de avanzar hacia una mayor atención a los resultados de aprendizaje como medida de calidad. Seguidamente, se recoge una síntesis de las principales críticas recibidas por las AGC. En último lugar, se presentan las conclusiones del análisis, que validan la hipótesis de la creciente convergencia internacional de los sistemas de educación superior.

\section{Conceptualización de las agencias de garantía de calidad, orígenes y evolución}

Las AGC no fueron una respuesta inmediata a la creciente atención a la regulación de calidad en la ES. Las tareas de aseguramiento y garantía de la calidad (GC, en adelante) fueron originalmente realizadas por una variedad de organizaciones, afiliadas a asociaciones gubernamentales o universitarias. El surgimiento de agencias independientes de GC se produce en un cambio en el modelo dominante de gobernanza del sector público. Hay dos razones principales para crear agencias, es decir, organismos reguladores autónomos en el sector público. En primer lugar, ayudan a separar la política de la administración (Lægreid \& Verhoest, 2010). Además, los organismos reguladores pueden considerarse más creíbles debido a su independencia de los políticos. En segundo lugar, se considera que las agencias son un mecanismo para lograr una mayor especialización, lo que se considera conducente a una mayor eficiencia. Se asume que disfrutar de un mayor grado de libertad conduce a una gestión más eficiente, debido a los beneficios de la especialización, profesionalización, flexibilidad, transparencia y apertura a las partes interesadas (Pollitt et al., 2001).

Las AGC fueron moldeadas por dos influencias diferentes. Una fuente de inspiración muy importante fue la existencia de tradiciones duraderas de acreditación de programas y universidades por parte de agencias privadas. Estas tradiciones fueron particularmente influyentes en los Estados Unidos, donde los organismos de acreditación de universidades se establecieron a fines del siglo XIX. Por otro lado, desde fines de la década de 1980, hubo una expansión mundial del modelo de agencia independiente como un nuevo diseño institucional capaz de mejorar la credibilidad de las decisiones en muchas áreas diferentes de las políticas públicas. Las agencias reguladoras se extendieron a un gran número de países y a nuevas áreas de regulación, como el suministro de servicios públicos o el control de riesgos sociales. Transformaron la estructura de las administraciones públicas en todo el mundo, proporcionando un nuevo marco para la expansión de las políticas reguladoras en diferentes sectores. El alcance global de la difusión de estas agencias fue impresionante. Llegaron a múltiples sectores de servicios públicos (electricidad, agua, saneamiento, telecomunicaciones, carreteras, ferrocarriles, puertos y aeropuertos, finanzas y salud, entre otros), creando presiones isomorfas que 
se generalizaron, y también se transportaron de un sector a otro de la actividad pública (Jordana, Levi-Faur, \& i Marín, 2011).

Una verdadera fiebre de «agencificación» caracterizó las reformas del sector público en muchos países europeos a partir de la década de 1990. Se esperaba que las agencias aumentaran el nivel de experiencia y profesionalidad de los reguladores, los hicieran más efectivos separándolos de la formulación de políticas y aumentaran la legitimidad de la regulación a los ojos de los regulados (Lægreid \& Verhoest, 2010). Surgieron en muchos países como organismos públicos autónomos, establecidos por los gobiernos para desarrollar e implementar nuevos instrumentos para la ES. Esta transformación institucional fue impulsada por la formulación de políticas y la financiación ofrecidas por instituciones globales como el Banco Mundial o la UNESCO, y la justificación se basó en la necesidad de separar a los proveedores de ES de los supervisores de calidad para otorgar credibilidad al nuevo sistema. En la medida en que muchos países tenían una gran cantidad de universidades públicas, el establecimiento de agencias separadas surgió como una solución para evitar interferencias políticas en la evaluación de calidad de la ES. La difusión de estas características de agencia también fue facilitada por el establecimiento de redes globales y regionales de agencias de aseguramiento de la calidad, como INQAAHE (Red Internacional para AGC en ES) o ENQA (Red Europea de Agencias de Calidad) (Blackmur, 2010).

Desde finales de la década de 1980, el modelo de AGC (AGC) centrado en la supervisión de las instituciones de educación superior (IES, en adelante) - separadas del gobierno - fue adoptado progresivamente por muchos países, como puede verse en la Ilustración 1.

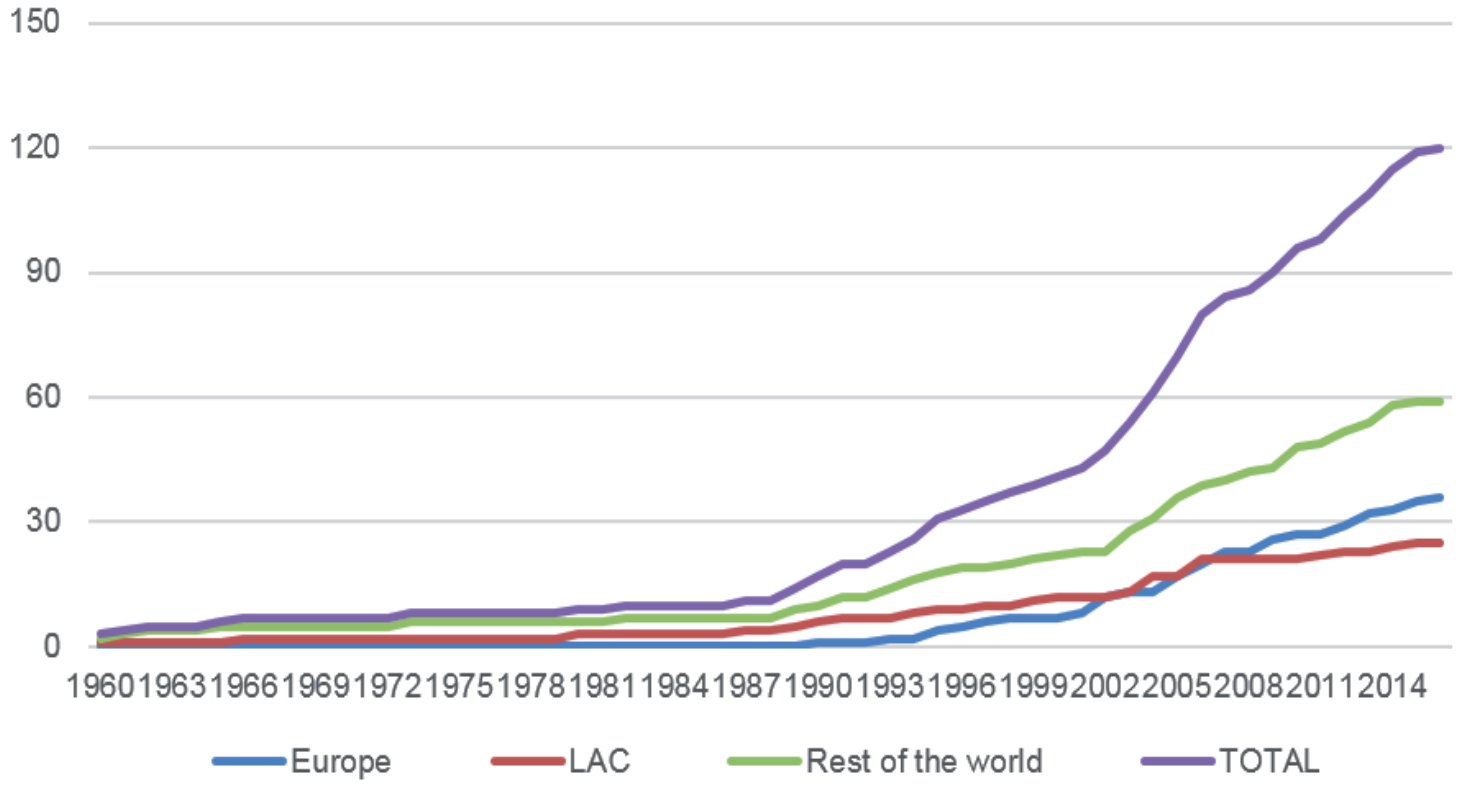

Figura 1. Fecha de creación de AGC en ES en Europa, América Latina y el Caribe y el resto del mundo. Fuente: Jordana, 2020.

Se han producido tres olas principales de desarrollo de GC de la ES a nivel mundial (Woodhouse, 2013). Las primeras agencias externas de GC se desarrollaron en los Estados Unidos a instancias de instituciones y asociaciones profesionales, en gran 
medida fuera del interés y la dirección del gobierno. Este modelo sigue siendo una rareza en el panorama mundial, ya que los esfuerzos posteriores en otros países fueron dirigidos principalmente por los respectivos gobiernos. Si la primera ola de control externo de calidad se produjo en Estados Unidos, la segunda llegó en la década de 1960 cuando gobiernos como el de Reino Unido e Irlanda establecieron agencias de control externo de calidad, no en respuesta a las preocupaciones del sector, sino para ayudar a guiar el desarrollo de un sector de rápido crecimiento y diversificación. La tercera ola de control externo de calidad comenzó en la década de 1990, cuando se desarrolló una explosión de agencias en todo el mundo. Como evidencia de tal crecimiento, en 1991 una docena de agencias de control de calidad de diferentes partes del mundo formó la INQAAHE.

En la década de 2010, casi todos los países habían creado sus propias agencias para supervisar la calidad de la ES, la mayoría separadas formalmente en el aspecto ejecutivo de sus ministerios padres. En solo tres décadas, la GC externa se ha desarrollado hasta convertirse en un campo regulatorio bien institucionalizado (Don F. Westerheijden, Stensaker, \& Rosa, 2007). Las AGC se han establecido prácticamente en todo el mundo, realizando actividades de acreditación, evaluación y auditoría de diferentes maneras y regularidades, y estableciendo múltiples redes internacionales. En Europa, el Proceso de Bolonia condujo a la adopción de un diseño de gobernanza para las AGC que enfatiza su independencia de los responsables políticos y también de las universidades. Por lo tanto, una AGC debe ser capaz de demostrar que la «definición y operación de sus procedimientos y métodos, el nombramiento de expertos externos, y la determinación de los resultados de sus procesos de GC se llevan a cabo de forma autónoma e independiente de los gobiernos, instituciones educativas y órganos de influencia política» (ENQA 2005).

\section{Modelos alternativos de gobernanza}

La evolución de la política de ES en las últimas tres décadas ha suscitado un renovado interés por la calidad. Si la eficiencia fue la palabra clave de la década de 1980, la calidad lo en la década de 1990. La rápida expansión del número de estudiantes, la búsqueda general de mejores servicios públicos y la creciente competencia entre las IES por los recursos y los estudiantes han suscitado un renovado interés por la calidad (Green, 1994). Paralelamente, la expansión de la ES transnacional o transfronteriza en esos años también suscitó demandas de GC (Stella, 2006). Por último, en la década de 2000, las nuevas modalidades de la ES, como el desarrollo de las tecnologías educativas, plantearon nuevos retos a la regulación de la ES. Como resultado de estos cambios, se ha argumentado que las IES se encuentran en una fase de transición en la que «la legitimidad de su misión, organización, funcionamiento, fundamento moral, formas de pensamiento y recursos se ponen en duda y se cuestionan» (Olsen, 2007).

Las configuraciones de las agencias indican tres enfoques de gobernanza: gobernanza externa, co-gobernanza o enfoque híbrido, y autorregulación (Jarvis, 2014). Como reguladores externos, las agencias asumen el papel de evaluadores de las instituciones, aportando perspectivas externas de examen de la labor realizada por las instituciones. Ejemplos de estos sistemas son Argentina, Australia, Colombia, México y Estados Unidos. México se clasificaría bajo la categoría de gobierno externo en términos de acreditación de títulos. La co-gobernanza se observa en Australia, Canadá, Corea del Sur y recientemente en el Reino Unido, donde se ha pasado de la autorregulación a la co-regulación. Australia y Corea del Sur tienen un riguroso marco de acreditación institucional, pero un sistema 
descentralizado de acreditación de títulos. El Reino Unido lleva a cabo un proceso formal de registro institucional, vinculado a mecanismos de financiación, y un proceso único de acreditación de títulos o "Degree Awarding Powers (DAPS)" que evalúa a la institución en su conjunto sin visitas externas. La posición de España es difícil de clasificar. Por un lado, sus nuevas políticas, como el programa AUDIT, inspiran a las instituciones a autorregularse. Por otro lado, la regulación nacional de acreditación es particularmente detallada y prescriptiva. Chile solía adoptar el autogobierno como modelo, sin embargo, esto podría cambiar con la reforma en curso de la ES.

$\mathrm{Al}$ analizar las diferentes racionalidades y prácticas, se puede explorar la manera en que las agencias pueden estimular la autorregulación del sector, dependiendo de su autonomía y orientación. Esto no es fácil porque las agencias tienden a definir criterios detallados y homogéneos para la acreditación. El caso de Canadá es un buen ejemplo en el que hay menos supervisión sobre el proceso, debido a la confianza pública tanto en el papel del gobierno como en el papel de las universidades en el diseño de sus propias regulaciones para títulos institucionales. Una pregunta importante, en este contexto, es: ¿puede la autorregulación crear las bases para la evaluación comparativa o, incluso, puntos de referencias dentro del propio sistema? En caso negativo, ¿̇están los sistemas preparados para asumir otros criterios relacionados con los mecanismos de financiación institucional que no tengan en cuenta el aseguramiento de la calidad?

Tabla 2.

Análisis comparativo: proceso de acreditación institucional.

\begin{tabular}{ll}
\hline Aspectos & Países \\
\hline $\begin{array}{l}\text { Solicitud preliminar previa a la } \\
\text { solicitud formal. }\end{array}$ & Australia, Chile, Colombia, algunas agencias en Estados Unidos. \\
\hline Auto-evaluación & $\begin{array}{l}\text { Argentina, Australia, Canadá/Ontario, Chile, Colombia, México, } \\
\text { Corea del Sur, España, Estados Unidos }\end{array}$ \\
\hline Evaluación externa & Todos excepto México, España y Reino Unido \\
\hline Estándares definidos centralmente & $\begin{array}{l}\text { Argentina, Australia, Canadá/Ontario, Chile, Colombia, Corea } \\
\text { del Sur, España, Reino Unido }\end{array}$ \\
\hline Costo: Gratis & Argentina, Colombia, España, México \\
\hline Costo: Recuperado & $\begin{array}{l}\text { Australia, Chile, Canadá, Corea del Sur, Reino Unido, Estados } \\
\text { Unidos }\end{array}$ \\
\hline Obligatorio & Argentina, Australia, Chile, Canadá/Ontario ${ }^{1}$, México, España \\
\hline Voluntario & Colombia, Corea del Sur, Reino Unido ${ }^{2}$, Estados Unidos \\
\hline
\end{tabular}

Fuente: Elaboración propia.

1 Las universidades de Ontario han acordado ejecutar procesos de auditoría cada ocho años.

2 No es obligatorio, pero las instituciones deben estar «registradas» para acceder a los fondos públicos. 
La mayoría de las agencias presentan una convergencia internacional con respecto a los mecanismos de GC. La gran mayoría incorpora los mecanismos de autoevaluación y evaluación externa por parte de sus pares y evoluciona con los cambios requeridos por las políticas nacionales. En otras palabras, participan de un lenguaje global común en cuanto a la GC.

Las principales convergencias en términos de acreditación institucional están relacionadas con el uso de la autoevaluación como mecanismo de acreditación, la definición central de estándares y la voluntad de resarcirse de los costes y de autofinanciarse. En la Tabla 2 se presenta un panorama general de análisis con referencia a las agencias estudiadas.

Las agencias analizadas acostumbran a mostrar una alta dependencia del gobierno y una menor dependencia de las instituciones. Las agencias no parecen interactuar con instituciones fuera del proceso de acreditación. Los gobiernos son la principal fuente de orientación sobre los estándares de GC, pero las agencias operan de manera independiente y en función del objetivo de autofinanciarse. Aunque muchas agencias incluyen académicos como parte de su cuerpo directivo, hay poca evidencia de interacción o diálogo con otras partes interesadas.

En definitiva, puede afirmarse que hay una gran variedad de maneras en que los países regulan la calidad académica, pero a pesar de esta variedad podemos ver una creciente convergencia en la estructura organizativa que los países utilizan para garantizar dicha calidad. Es evidente que la GC en ES se ha convertido en un ámbito reglamentario maduro en el que los organismos autónomos constituyen una esquina del triángulo regulador, junto con los responsables de las políticas (Parlamento, Gobierno) y las universidades. Por ejemplo, una gran mayoría de los países europeos depende de organismos de GC semiindependientes o formalmente autónomos. Una encuesta de la Asociación Europea para la GC (Costes et al.) indicó que el número de agencias independientes de calidad de la ES en Europa está aumentando constantemente; además, sus tareas se están ampliando y utilizan un número creciente de instrumentos de evaluación diferentes (ENQA, 2008). Esta forma de organización también está fuertemente promovida por las Normas y Directrices Europeas para la GC (ENQA, 2005). Como resultado, en Europa y en solo una década, países con puntos de partida y enfoques muy diferentes para la GC académica han adoptado modelos bastante similares de agencias reguladoras, prácticamente convergentes.

Pero más allá del continente europeo parece haber una gran convergencia en el resultado final a escala internacional, con algunas excepciones importantes como el Reino Unido. En efecto, la mayoría de las agencias son autorizadas por el Parlamento para que se encarguen de la GC. No se encuentran dentro de una estructura jerárquica del Ministerio, pero están vinculadas a este mediante una Junta que es designada por el Ministro a cargo de la ES. El Ministro también es responsable de supervisar el desempeño general de la agencia, requiriendo evaluaciones externas periódicas. Por supuesto, existen diferencias funcionales: algunas acreditan instituciones, no programas; algunas llevan a cabo las evaluaciones por sí mismas, mientras que otras dependen de asociados externos, etc., pero la estructura y los procedimientos de la organización son sorprendentemente similares. También parece existir un vínculo entre la introducción de un nuevo instrumento y la revisión de la estructura de las agencias existentes. Un sistema de evaluación más jerárquico (por ejemplo, la acreditación) requiere más autonomía de las organizaciones profesionales (por ejemplo, las reguladas), que una evaluación colegiada 
orientada a los pares. Si bien la cooperación con las asociaciones profesionales parece funcionar bien en el caso de evaluaciones tipificadas como más suaves, los instrumentos de mayor dificultad, como la acreditación o las evaluaciones formales de asignaturas, parecen requerir una mayor distancia, pero también un mandato jurídico más claro.

Sin embargo, varios estudios han cuestionado el alcance de la aplicabilidad de un modelo tan universal, argumentando que ha persistido una variación sustancial en la regulación de la GC, particularmente en relación con el perfil de las agencias y las características de los instrumentos implementados (Billing, 2004). Curiosamente, Brennan y Shah (2013) afirmaron que no todos los países tenían una agencia de GC (por ejemplo, Italia para entonces), y en algunos países no había una sola agencia sino muchas (por ejemplo, Canadá, Alemania, México, España, EE. UU.). En una línea similar, apoyada en nuevas comparaciones basadas en una ampliación del estudio de 1993, Billing (2004) mostró que no hay un «modelo general» de GC que se aplique universalmente, pero que la mayoría de sus elementos, particularmente los relacionados con sus métodos y procedimientos de trabajo, se encuentran en la mayoría de los países.

\section{La convergencia metodológica: de las revisiones a las auditorías}

En el sector de la ES, la mayoría de las agencias utilizan cuatro métodos básicos para la revisión de calidad, a saber, la autoevaluación seguida de una revisión realizada por pares y/o revisión externa, y auditorías. Desde una perspectiva general, la elección del enfoque de calidad (acreditación/evaluación/auditoría) pareciera no tener mayor influencia sobre los elementos metodológicos:

a) La autoevaluación es un elemento clave en la mayoría de los procedimientos de evaluación. Proporciona un estándar contra el cual la IES puede medirse y un marco para construir una definición de calidad. Por lo tanto, ayuda a la IES a verificar hasta qué punto está logrando su misión y objetivos estratégicos, y le permite preparar un plan de acción para un mayor desarrollo. Muchas IES llevan a cabo autoevaluaciones, aunque su naturaleza varía significativamente. En los Estados Unidos, ha habido una larga tradición de auto-revisiones (generalmente denominadas auto-estudios) en los procedimientos de acreditación y las IES tienden a tener una gran capacidad para la recolección, análisis e interpretación de información para tales procedimientos (Brennan, 2018). En Europa, se incluye una autoevaluación en el 94 \% de los procesos de evaluación y en el 68 \% de los procedimientos de acreditación (ENQA, 2003). Para facilitar la realización de autoevaluaciones, prácticamente todas las AGC europeas brindan orientación o manuales, aunque solo una minoría de ellas brinda capacitación (Thune, 2006).

b) Revisión por pares. Una revisión por pares es una evaluación realizada por otro académico o grupo de académicos, generalmente de la misma disciplina. Las revisiones por pares, que ya son dominantes en la evaluación de la investigación, también se utilizan cada vez más en la evaluación de programas y procesos de enseñanza y aprendizaje. Sin embargo, quién es considerado como un par varía en los diferentes sistemas de GC. En los procedimientos 
de acreditación de los Estados Unidos, las revisiones por pares involucran a docentes y colegas administrativos de la profesión y se llevan a cabo para revisar la autoevaluación y realizar visitas al sitio (Eaton, 2011).

c) Revisión externa. Cada vez más, los paneles de revisión de calidad incluyen miembros no académicos y personas de otros países además de sus pares (Woodhouse, 2003). En los Estados Unidos, por ejemplo, el panel de revisión también puede incluir miembros no académicos. En el sistema danés de GC hay examinadores externos permanentes y asalariados y el panel de revisión incluye no solo expertos profesionales o académicos, sino también representantes de los empleadores (Thune, 2006).

d) Auditorías. Las auditorías institucionales se centran en procesos para monitorear y mejorar la calidad en la institución. Para muchos, la auditoría es el instrumento superior que logra llegar hasta el núcleo de los procesos institucionales y respaldar eficazmente las acciones de colaboración dentro de la universidad para cambiar realmente la calidad de los procesos de enseñanza y aprendizaje (D. D. Dill \& Beerkens, 2010). Su atractivo puede explicarse no tanto debido a sus beneficios internos, sino por los costos sustanciales que las acreditaciones de programas imponen a los gobiernos y a las IES. Lo que no parece tan claro es si una auditoría institucional puede atender con la misma eficacia las necesidades de rendición de cuentas de los interesados externos.

La elección de un instrumento específico de GC está, por supuesto, fuertemente influida por las preocupaciones identificadas anteriormente: cuál es el problema de calidad percibido y, por lo tanto, cual es la definición implícita de calidad, y cuál, en definitiva, es el propósito principal de la GC.

Las quejas sobre la carga regulatoria han alentado a muchas agencias a buscar un enfoque metodológico más «ligero» para garantizar la calidad. Esta presión tiende a conducir hacia las auditorías institucionales como un enfoque dominante de GC. En el discurso europeo sobre la GC en ES, por ejemplo, una auditoría «es un proceso para verificar que existen procedimientos para garantizar la calidad, integridad o estándares de provisión y resultados». El Documento 14 de la Asociación Europea de AGC en ES (ENQA) brinda una definición aún más precisa: «Una auditoría es una evaluación de las fortalezas y debilidades de los mecanismos de calidad establecidos por una institución para monitorear y mejorar continuamente las actividades y servicios de una asignatura, un programa, toda la institución o un tema». El término auditoría en realidad solo se emplea en unos pocos países (Finlandia, Suiza y Austria), mientras que otros países usan una variedad de términos. En el Reino Unido, el término «auditoría» incluso ha sido tan dilapidado que ya no es utilizado por las AGC.

Hay un claro cambio hacia las auditorías institucionales en países como los Países Bajos, Austria y otros (Hopbach, 2012), En lugar de estándares, las auditorías institucionales se centran en los procesos institucionales para monitorear y mejorar la calidad en la institución. Las auditorías suelen tener tres características básicas:

a) Centrarse en asegurar la calidad institucional. Los procedimientos tienen un enfoque explícito en las medidas y/o procesos establecidos por las IES con el propósito de asegurar y desarrollar la calidad en la enseñanza y el aprendizaje, 
y (en algunos casos) en la investigación y el desarrollo. Las auditorías deben distinguirse de las revisiones institucionales, que van más allá de la GC interna y se refieren más a criterios relacionados con las ofertas de las instituciones, como programas de estudio, investigación, infraestructura y recursos.

b) Orientación de mejora. Las auditorías afirman apoyar la mejora de calidad en las IES. A diferencia de la acreditación, no verifican principalmente el logro de estándares (mínimos) con respecto al reconocimiento formal de un (nuevo) programa de estudio o institución, sino que demuestran la capacidad de una institución (existente) para garantizar (y desarrollar) su propia calidad.

c) Evidencia a través de muestras. Las auditorías exigen evidencia de la existencia de un control de calidad interno que suele proporcionarse a través de muestras, como «objetivos de auditoría», «temas seleccionados», «campos de rendimiento» o «muestras de programas de estudio». Estas muestras están destinadas a demostrar el rendimiento de la GC interna y se revisan con más detalle.

Una novedad importante en las últimas décadas es la introducción de evaluaciones y acreditaciones de asignaturas patrocinadas por el Estado. A diferencia de las acreditaciones especializadas en los Estados Unidos, estas evaluaciones son exhaustivas y cubren la mayoría de los campos de materias en los que los estudiantes universitarios están matriculados. Si bien emplean la revisión por pares, se llevan a cabo bajo los auspicios del Estado y, por lo tanto, están diseñadas para reflejar mejor el interés público en garantizar estándares académicos en lugar de los intereses especiales de las asociaciones profesionales.

Las evaluaciones de asignaturas desarrolladas en Dinamarca, los Países Bajos y el Reino Unido han contribuido de manera importante a mejorar la enseñanza, así como la estructura y el contenido de los currículos académicos en estos sistemas universitarios tradicionales en un número limitado de universidades, disciplinas y campos. Sin embargo, las evaluaciones temáticas son un instrumento menos eficaz para ampliar los sistemas con nuevos campos de estudio. Las acreditaciones generales de asignaturas, tal como se aplican en Alemania y en varios otros países influidos por las reformas de Bolonia, abordan mejor el desarrollo de nuevos campos y títulos, pero igual con lo que pasa con las evaluaciones de asignaturas, estos instrumentos de evaluación inter-pares son extremadamente intensivos en mano de obra, costosos en términos de tiempo y esfuerzo académico y, por consiguiente, insostenibles a la larga.

\section{La convergencia de las problemáticas}

A pesar de las notables diferencias entre los modelos que se acaban de esbozar, el análisis de las preocupaciones reflejadas en la literatura en cada uno de los casos demuestra, de nuevo, que existe una segunda convergencia que se añade a las de los métodos de trabajo: la de las problemáticas. Estas se giran en torno a tres ejes: la independencia política, la rendición de cuentas y la necesidad de un mayor énfasis en los resultados de aprendizaje, que se examinan a continuación. 


\subsection{La independencia política}

La independencia se ha definido como la capacidad de los organismos para decidir sobre cuestiones de su responsabilidad sin injerencias políticas (Jordana, García Juanatey, Pérez Durán, \& Sancho Royo, 2018) (Juanatey et al., 2020). Desde esta perspectiva, la independencia puede estar determinada por el alcance y extensión de las competencias decisorias de la agencia (Verhoest, Peters, Bouckaert, \& Verschuere, 2004). Quienes investigan la independencia de las agencias prestan atención a los mecanismos de control que tienen los decisores políticos sobre los órganos rectores de las agencias, específicamente sobre los miembros y los presidentes de los consejos de las agencias. Desde esta perspectiva, las principales preocupaciones se basan en el análisis de hasta qué punto las normas formales permiten a los organismos rectores tomar decisiones sin interferencias políticas, y medir cómo se definen los mecanismos de designación y renovación de dichos organismos para permitir un comportamiento autónomo. Esta probablemente sea la dimensión más relevante para la independencia de la agencia.

El modelo de agencias independientes de GC a menudo se promueve a partir de una justificación muy específica del sector de la ES. La GC debe ser independiente del control político para garantizar su legitimidad a los ojos de las universidades, y debe ser independiente de las universidades para evitar la «captura regulatoria» (Beerkens, 2016). Sin embargo, la tendencia hacia la independencia de las agencias reguladoras es una instancia más de la misma tendencia en otros sectores de la actividad pública.

En la ES europea, la búsqueda de la independencia ha sido bastante generalizada y promovida como uno de los estándares más reconocidos por las agencias. De hecho, de acuerdo con las Normas y Directrices para la GC en el Espacio Europeo de ES (ESG), adoptado en 2005, una AGC debe poder demostrar que: «la independencia operativa de las IES respecto de los gobiernos está garantizada en la documentación oficial (por ejemplo, en los instrumentos de gobernanza o normas legislativas) y que la definición y el funcionamiento de sus procedimientos y métodos, la designación y el nombramiento de expertos externos y la determinación de los resultados de sus procesos de GC se llevan a cabo de forma autónoma e independiente de los gobiernos, las IES y otros órganos con eventual influencia política' (ENQA 2005). Desde entonces, el ESG se ha implementado con bastante éxito en agencias que pertenecen a 46 países (Alzafari \& Ursin, 2019) y la independencia de las agencias se ha convertido en un rasgo de identidad muy relevante en la mayoría de las AGC.

Algunas investigaciones empíricas recientes han demostrado que las diferencias entre las agencias europeas y latinoamericanas no son muy significativas (Jordana et al., 2018) en lo que se refiere a la independencia y la autonomía política. Por ejemplo, con respecto a la autonomía de los directores y miembros de la junta de las agencias, los resultados promedio en cada región son bastante similares. En cuanto a la rendición de cuentas ante el ejecutivo, las agencias latinoamericanas son ligeramente menos fiables que las europeas. Sin embargo, el resultado promedio en cuanto a autonomía política es el mismo, como se muestra en la Ilustración 2. 


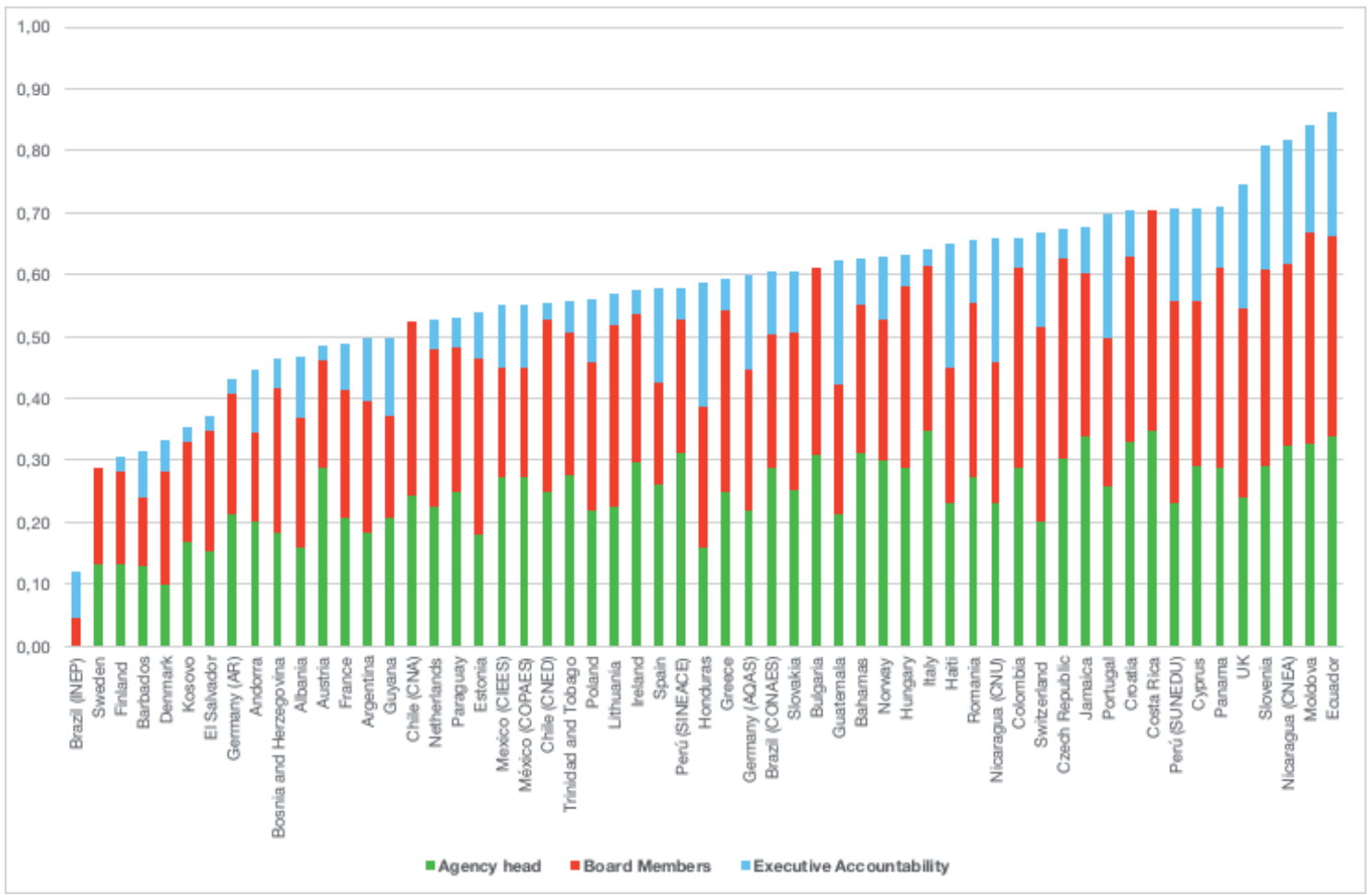

Figura 2. Clasificación de las AGC en la ES en Europa y América Latina según la autonomía política del gobierno correspondiente. Fuente: Jordana, 2020.

Los resultados muestran variaciones significativas en las diferentes agencias y países, aunque también se observan signos de convergencia de manera bastante visible. En cuanto a los casos europeos, mientras que algunas agencias de Europa occidental, como las de los países escandinavos, como Dinamarca o Finlandia, muestran puntuaciones bajas, otras agencias, como las del Reino Unido, Croacia o Eslovenia, tienen AGC con niveles más altos de independencia política en nuestro índice. En América Latina, también se observan casos de autonomía política significativa; por ejemplo, en Chile, Perú o Ecuador.

Sin embargo, es importante destacar que el hecho de que una agencia tenga un nivel significativo de independencia con respecto al poder político no significa una mayor independencia de las universidades. Por el contrario, en varios casos, particularmente en América Latina, las universidades parecen tener un papel decisivo en la toma de decisiones de las AGC (universidades públicas, en algunos casos). En consecuencia, las AGC probablemente estén bien protegidas de la interferencia política, pero no siempre tienen, al mismo tiempo, medidas preventivas para evitar la participación directa de las universidades en la toma de decisiones para defender sus propios intereses y puntos de vista sobre cómo deben ser gestionados y desarrollados los procedimientos de GC. La evidencia muestra que algunas AGC son formalmente más distantes del gobierno, a menudo más estrechamente vinculadas a las asociaciones universitarias que al gobierno central y su personal no es necesariamente funcionario público (Beerkens, 2016). Este fenómeno es conocido como «captura regulatoria» (Baldwin, Cave, \& Lodge, 2011) expresión con la se quiere indicar que la regulación puede servir más a los intereses de los regulados que al interés público. Una simple argumentación afirmaría que las agencias sirven a los intereses de las universidades debido a sus fuertes vínculos con ellas, a través de la experiencia, la movilidad profesional, las interacciones comunes, la historia compartida, etc. 
No hay mucha evidencia empírica disponible sobre el beneficio de la autonomía en el caso de las AGC en ES. Según las Normas y Directrices citadas anteriormente, parece que la principal preocupación es la objetividad del proceso, la cual requiere independencia tanto de los políticos como de las universidades. Ewell (2010) es uno de los pocos que ha analizado las ventajas de las agencias autónomas en el aseguramiento de la calidad. En el contexto de los Estados Unidos, ve dos razones principales por las cuales las agencias independientes son más efectivas que la intervención estatal directa. Los estados se ven severamente amenazados por la escasez de recursos y, por lo tanto, no pueden apoyar programas de calidad suficientemente amplios, o financiar programas basados en el rendimiento. En segundo lugar, en un contexto de agenda política a corto plazo y severo partidismo, sería difícil mantener una agenda política consistente a largo plazo. Si bien las iniciativas estatales sólidas, como la financiación basada en el desempeño, demostraron ser de corta duración y no tuvieron mucho impacto en los niveles superiores de la gestión universitaria, presionar a las agencias de acreditación para que sigan la agenda de los gobiernos parece haber sido mucho más eficaz. Cuando el Gobierno Federal aumentó gradualmente la presión sobre las organizaciones de acreditación para que se centraran en los resultados del aprendizaje de los estudiantes, lo cual se reflejó cada vez más en sus exámenes, la mayoría de las instituciones habían desarrollado a finales del decenio de 1990 el tipo de infraestructura de evaluación originalmente prevista, pero no cumplida por los mandatos estatales. Por lo tanto, parece ser mucho mejor distanciar la GC de la política, no solo para tener legitimidad, sino también para mostrar un compromiso creíble.

La tensión entre la autonomía y la rendición de cuentas, o la desregulación y la reglamentación, es un problema constante en muchos sectores de la administración pública. El sector de ES puede tener cierta especificidad, debido a la noción de libertad académica y desconfianza histórica en la intervención gubernamental, pero el dilema autonomíarendición de cuentas es, sin embargo, muy visible. Aunque la mayoría de las agencias suelen afirmar que tienen autonomía sobre los procedimientos y decisiones de revisión, la realidad es que están estrechamente afiliadas al gobierno debido a sus acuerdos de financiación, que por lo general dependen casi por completo de la financiación pública. Los organismos autofinanciados y privados son más autónomos que los públicos y los cuasi-gubernamentales, debido a su financiación y recursos independientes. De ahí la creciente tendencia a la autofinanciación como un mecanismo adicional de preservación de la independencia que, al mismo tiempo, conviene a los objetivos políticos de reducción del gasto público.

Varios especialistas han expresado su preocupación por esta cuestión. Brown (2013) ha afirmado que cuando el gobierno desarrolla iniciativas de GC como parte de las estrategias de reforma de la ES, su intervención en el diseño de la GC se vuelve inevitable. Ewell (2010) también señaló que conseguir que el gobierno apoye el proceso de GC sin perder la autonomía de la agencia o afectar su funcionamiento es, sin duda, una opción a considerar. Dill (2011) indicó que incluso las agencias de auditoría o reconocimiento gubernamentales verdaderamente independientes muestran variaciones sustanciales en subjetividad y rigor. El organismo de acreditación puede sentirse obligado a cumplir los deseos del gobierno en lugar de los sugeridos por su propia agenda y misión. Esto sugiere que la autonomía e independencia de la agencia se vería amenazada en la medida en que el gobierno mantenga el control burocrático de su gobernanza interna y la revisión externa. Para enfrentar este dilema, las AGC deben ser más responsables de la calidad de su trabajo y están obligadas a demostrar que su profesionalidad no se verá afectado por este control externo. 


\subsection{La rendición de cuentas: ¿quién evalúa a las agencias de evaluación?}

A medida que aumenta la preocupación pública por la responsabilidad de los organismos de GC, se espera que demuestren a los interesados que se toman en serio las actividades de acreditación y que tienen un impacto positivo en la ES. Las AGC deben ser autocríticas, objetivas y de mente abierta (Costes et al., 2010) al chequear la calidad de las IES. Expectativas como esta han llevado a las AGC a reconocer el examen externo como un ejercicio legítimo a nivel nacional e internacional. Esto se conoce como «rendición de cuentas de la acreditación». Eaton (2011) afirma que la responsabilidad de las AGC se refiere a cómo, y en qué medida, estas agencias aceptan su responsabilidad por la calidad y los resultados de su trabajo y responden abiertamente a los constituyentes y al público. Por lo tanto, se espera que los organismos de GC adopten diversos enfoques internos y externos para demostrar la calidad de sus actividades de revisión (Woodhouse, 2010).

Las limitaciones documentadas de los actuales enfoques estatales y auto-reguladores de la garantía externa de calidad académica han motivado a muchos países a establecer nuevos organismos nacionales de GC académica, como el Organismo de Calidad de las Universidades Australianas, el Instituto Danés de Evaluación, el Consejo Alemán de Acreditación y la Agencia de GC (Reino Unido). En el diseño de estas agencias, se han hecho serios esfuerzos para garantizar que reflejen el interés público más amplio, al estructurarlas, en la medida de lo posible, para que sean independientes tanto de las universidades como del gobierno. Pero debido a que a estas agencias se les otorga autoridad pública para diseñar, conducir y supervisar los procesos externos requeridos de GC, son esencialmente reguladores gubernamentales que generan sustanciales costos directos importantes para el erario público, así como costos indirectos para el sector universitario.

Estados Unidos fue el primer país en desarrollar un sistema de reconocimiento de calidad de las AGC a nivel nacional. En 1998, el Consejo para la ES (CHEA), la organización de acreditación estadounidense, anunció sus estándares para el reconocimiento de los acreditadores estadounidenses (CHEA 2006). Alemania también desarrolló un esquema de reconocimiento en una etapa temprana, esencialmente debido a su sistema de acreditación descentralizado. Las agencias alemanas de GC deben estar acreditadas por el Consejo de Acreditación de la Fundación para la Acreditación de Programas de Estudio, que actúa como el órgano central de toma de decisiones de la fundación para definir los requisitos básicos del proceso y se encarga de que cualquier acreditación se lleve a cabo sobre la base de criterios confiables, transparentes y reconocidos internacionalmente. El sistema de reconocimiento internacional para las AGC se inició en Europa con el propósito de la integración regional en la ES. En 2005, la Asociación Europea para el Aseguramiento de la Calidad en la ES (ENQA) declaró que sus requisitos para una membresía completa implicarían alcanzar la parte III de las Normas y Directrices para el aseguramiento de la calidad en la ES del Área Europea.

Por lo general, los propios organismos adoptan varios enfoques internos de GC, como la recopilación de testimonios de las instituciones examinadas mediante encuestas de satisfacción y la celebración de reuniones, seminarios y congresos formales o informales con las partes interesadas. Recientemente, algunas agencias han organizado grupos de discusión focalizados y han realizado meta-evaluaciones para reunir información relevante para su propia revisión. Estas diferentes herramientas para garantizar la calidad interna pueden tener sus ventajas y sus inconvenientes. Las encuestas anónimas, por ejemplo, tienden a ser positivas y no permiten una mayor interacción con los encuestados. 
Además, es probable que las nuevas ideas que surjan no provengan de la institución que se está evaluando. Por el contrario, los grupos de discusión focalizados parecen ser una forma más rápida y eficiente de recabar opiniones. Sin embargo, su limitado número de participantes hace que sea difícil determinar si toda la información que producen es precisa e imparcial. En respuesta a ello, las AGC han comenzado a adoptar múltiples métodos para obtener información desde las perspectivas macro y micro.

$\mathrm{El}$ aseguramiento interno de la calidad es un elemento importante para el aseguramiento de la calidad de las AGC. Sin embargo, un estudio de la Asociación Europea para la GC en la ES (ENQA) que revisa los informes de autoevaluación de 34 agencias europeas de GC encontró que solo el 65 por ciento de las agencias cumplen con los estándares de rendición de cuentas. Concluyó que la implementación del mecanismo interno de GC está todavía en fase de desarrollo. Pero su llegada ya ha traído consigo varios impactos en las AGC, como el conocimiento interno de la propia organización, la creación de una metodología para controles internos y la conciencia de las debilidades y fortalezas de una organización (Costes et al., 2010).

Las revisiones externas consisten en un proceso que utiliza personas externas al programa o institución para evaluar la calidad o los estándares. En otras palabras, una revisión externa de calidad realizada por un tercero, que podría ser un gobierno, un organismo de reconocimiento o una red internacional, tiene por objetivo reconocer la calidad de las actividades de la agencia. Hoy en día, el cambio de la mejora interna hacia la responsabilidad externa parece prevalecer entre las AGC. De hecho, varias AGC comenzaron a principios de la década de 1990 a examinarse a sí mismas a través de mecanismos de revisión externos, como la Middle States Association of Schools and Colleges en Estados Unidos, el Higher Education Quality Council en Reino Unido, el University Grants Committee en Hong Kong, el Akkreditierungsrat en Alemania, y el North-Central Association of Schools and Colleges en EE. UU. Las principales razones para la revisión externa incluyen la necesidad de renovar el estatuto de su reconocimiento público, ayudar en el desarrollo de los planes y actividades futuras de la agencia y demostrar que cumple con las expectativas de las partes interesadas.

Las redes de GC han comenzado a revisar las AGC externamente a través de un conjunto de criterios con la finalidad de otorgar o mantener la membresía. La Asociación Europea para el Aseguramiento de la Calidad en la ES (ENQA) es la primera organización en revisar externamente el estado de todos sus miembros, incluidos sus procedimientos de GC para la ES, el estatuto oficial, las actividades, los recursos, la declaración de misión, la independencia, los criterios y el proceso, y sus mecanismos de rendición de cuentas. Hace hincapié en que esta revisión externa debe ser objetiva y debe proporcionar evidencia suficiente para determinar si la agencia cumple con los estándares europeos para las agencias externas de GC (ESG) y, por lo tanto, con los requisitos de membresía estipulados por ENQA. En 2009, INQAAHE realizó una revisión de la adhesión de sus miembros a las Directrices de Buenas Prácticas de INQAAHE. A diferencia de la ENQA, la revisión externa es aplicada voluntariamente por sus miembros. Los estándares de revisión incluyen la gobernanza, los recursos, la GC de la agencia, la presentación de reportes de información pública, la relación con las instituciones, los estándares y las revisiones internas para las instituciones, los sistemas de evaluación, decisión y apelaciones, la colaboración con otras AGC y las políticas sobre la ES transfronteriza. Para la revisión, utiliza un panel de expertos internacionales quienes realizan una visita in situ. Se presenta a las agencias revisadas un informe final que indica las áreas específicas en las que se observan las deficiencias para las acciones de seguimiento. 


\section{3. ¿Evaluar la calidad sin evaluar los resultados de aprendizaje?}

Hasta hace muy poco, los sistemas de calidad se habían centrado en insumos y procesos, y con menos frecuencia en productos y resultados. Los sistemas externos de GC en ES al principio se circunscribieron a la medición de insumos; por ejemplo, el nivel de calificación del personal docente y el número de libros en la biblioteca, como forma de medir la calidad de la prestación del servicio. Aunque las mediciones de insumos suelen ser objetivas y de fácil acceso, por sí solas proporcionan poca evidencia de la calidad en la práctica. El hecho de que un miembro del personal docente tenga una maestría o un doctorado podría considerarse importante y tal vez necesario, pero no es suficiente para garantizar su capacidad como docente. La financiación es otro insumo. Podría suponerse que una financiación adecuada es una condición previa para crear un entorno de aprendizaje eficaz. Sin embargo, definir qué nivel de financiación sea el adecuado es a menudo difícil y controvertido y, una vez definido, un financiamiento adecuado no es, en sí mismo, garantía de calidad.

Los procesos, en particular los métodos de enseñanza y evaluación, pueden proporcionar una indicación de la probable eficacia que tiene la experiencia de aprendizaje para los estudiantes. Es por esta razón que son supervisados en muchos sistemas de GC en ES. Por ejemplo, los procedimientos para la calificación externa de las evaluaciones y exámenes escritos pueden tomarse como indicadores de la fiabilidad de los procesos de evaluación y, por lo tanto, de la validez de los resultados y calificaciones otorgados por un determinado programa. Sin embargo, muchos aspectos de la enseñanza y del aprendizaje son difíciles de captar y evaluar de manera binaria o cuantitativa, dificultando la recopilación de datos cuantitativos sobre esos procesos.

En los últimos años, se ha puesto cada vez más énfasis en los productos, tales como las competencias de aprendizaje (Stensaker, 2014). Los resultados como «unidad de análisis» tienen cierto atractivo. Este enfoque transmite la sensación de que el sistema de GC está tratando de captar lo que realmente importa: se centra en lo que el proceso produce y consigue y no se preocupa tanto por los mecanismos del proceso en sí mismos. Las mediciones de los resultados también adoptan formas muy diferentes. Suecia es un ejemplo de un sistema que abandonó por completo las mediciones de los procesos de evaluación y se centra en la calidad de la tesis final de los estudiantes. También se han intentado crear exámenes nacionales de grado, lo que nuevamente apunta hacia la medición directa de los conocimientos y competencias de los estudiantes al final de sus estudios. Las mediciones basadas en los resultados son conceptualmente atractivas, particularmente entre los interesados externos. Sin embargo, todos los intentos parecen fracasar debido a problemas de fiabilidad y validez del instrumento y a las sensibilidades políticas. Las mediciones del valor agregado en ES parecen seguir en la búsqueda del santo grial que al final puede distorsionar o disminuir los estándares académicos en lugar de asegurarlos (D. D. Dill \& Beerkens, 2010).

Los productos más directos del proceso educativo son graduados con mayores conocimientos y habilidades (resultados de aprendizaje) adquiridos a través de su educación superior. Aislar el valor agregado específico de la experiencia de ES de un estudiante de otros factores como el contexto social y cultural es intrínsecamente muy difícil. Sin embargo, a nivel internacional, en algunos países se utilizan pruebas estandarizadas para medir las capacidades y competencias de los estudiantes y graduados de ES de manera comparable. 
En Estados Unidos, por ejemplo, las pruebas CLA y CLA+ se han desplegado ampliamente para examinar competencias genéricas (CAE, 2018), mientras que, en México, los Exámenes Generales para el Egreso de Licenciatura (Siegel) son utilizadas por muchas instituciones para evaluar a los graduados en áreas disciplinarias específicas (CENEVAL, 2018). En Colombia, todos los estudiantes de ES están obligados a realizar una prueba de competencia general (Saber Pro) para poder graduarse (ICFES, 2018). Aunque los exámenes Saber Pro en Colombia son obligatorios, los resultados obtenidos por los estudiantes no se utilizan directamente para generar puntajes de calidad para las instituciones a las que asistieron. El examen ENADE en Brasil es el único ejemplo de exámenes externos a gran escala que son obligatorios para los estudiantes y a la vez son utilizados directamente en la GC de los programas e instituciones.

Otras medidas relacionadas con el producto o con los resultados no toman en cuenta el aprendizaje de los estudiantes per se, sino más bien cuestiones indirectamente relacionadas con él. El concepto de producto toma entonces en cuenta las tasas de graduación y compleción (por ejemplo, proporción de estudiantes que completan con éxito su curso). En los resultados se incluyen cada vez más factores indicativos de la empleabilidad (por ejemplo, la proporción de graduados que están empleados y en qué tipos de trabajo).

La relación entre las mediciones del producto y los resultados, por un lado, y la calidad de los cursos y programas, por otro, no siempre es sencilla. Si bien puede esperarse que un curso de alta calidad brinde un buen apoyo a los estudiantes de diferentes orígenes para permitirles completar su curso con éxito, una tasa de finalización del $100 \%$ puede ser una indicación de bajos estándares, en lugar de serlo de buena calidad. Si bien es lógico suponer que los cursos de alta calidad preparan bien a los estudiantes para obtener buenos trabajos, los resultados del empleo de graduados dependen de una amplia gama de factores más allá de la calidad del programa educativo. Graduados de un curso prestigioso, pero objetivamente de mala calidad, pueden tener éxito en la obtención de buenos trabajos. Los graduados de cursos de alta calidad pueden tener dificultades para encontrar un trabajo adecuado si la relación entre el contenido de los cursos y los conocimientos y habilidades requeridos en el mercado laboral es débil o las condiciones generales de empleo son difíciles.

\section{Las críticas a las agencias de garantía de la calidad}

La AGC en ES se ha convertido en un campo regulatorio maduro. Las AGC en ES son elogiadas por su legitimidad, experiencia y compromiso creíble. Al mismo tiempo, es importante tomar consciencia de las debilidades que pueda mostrar el modelo predominante de agencia. En determinadas circunstancias, la agencificación puede conducir a instrumentos de política fragmentados y descoordinados, puede llevar a una aproximación técnica a la GC basada en la experiencia, que esté desligada de la dirección política y puede plantear cuestiones de responsabilidad a los ojos del público. Lo más importante es que las agencias se han convertido en un actor central en la regulación de calidad en ES y, por tanto, en un actor con identidad e intereses estratégicos propios (Verhoest et al., 2004).

La creación de AGC en ES ciertamente ha llevado a una fragmentación importante: ahora es más difícil coordinar las actividades de las agencias independientes donde existen varias. En muchos países existen diferentes instrumentos de evaluación. Las 
universidades han tenido que dar entrada a varias visitas in situ, proporcionar datos para múltiples esquemas y organizaciones de evaluación, y suministrar información a diferentes organizaciones. El problema de la fragmentación es quizás más visible en el Reino Unido y se ha abordado explícitamente mediante cambios en las políticas. Se estableció una nueva agencia de GC para combinar todas las herramientas de evaluación, anteriormente bajo dos organizaciones diferentes. Los problemas actuales de fragmentación son abordados por un comité permanente cuya tarea es garantizar la coherencia y ofrecer sugerencias de mejora si es necesario. Por otro lado, es difícil afirmar si los problemas de fragmentación y la sobrecarga de evaluación tienen su origen en la independencia de las agencias. Las tensiones parecen surgir a menudo del hecho de que las universidades enfrentan diferentes demandas de diferentes grupos de partes interesadas: se espera que la garantía externa de calidad garantice estándares mínimos, pero también que funcione como una herramienta de transparencia y, al mismo tiempo, debe ofrecer incentivos para mejorar la calidad interna garantizando la legitimidad política (Beerkens, 2018).

El segundo problema genérico de la agencificación se refiere al debilitamiento del núcleo político: los encargados de formular políticas no pueden dirigir a los organismos independientes tan estrechamente como lo hacen con sus propios departamentos ministeriales. Esto también puede crear problemas de rendición de cuentas. Los encargados de la formulación de políticas pueden tener la impresión de que pierden el control, ya que el público los considera responsables de los problemas pero se supone que no deben interferir en las actividades de las agencias.

En los Países Bajos, un escándalo de calidad planteó preguntas sobre el papel de la agencia autónoma frente al papel de la inspección ministerial. Aunque la agencia no era responsable de los problemas, las acciones y las propuestas dieron la impresión de que el ministerio percibía la necesidad de una fuerza más operativa, dando paso a la creación de su propia inspección de ES. Sin embargo, tampoco esto no estuvo exento de críticas y desafíos.

Por un lado, se ha argumentado que la abundancia de iniciativas de GC, con diferentes procedimientos de evaluación, acreditación y recopilación de datos, puede causar fatiga entre las universidades, ya que estas tienen que informar continuamente sobre las actividades realizadas a organismos externos (Don F Westerheijden, 2007). Por otra parte, también debe notarse la creciente actividad de los reguladores privados globales, como los productores de rankings, liderados por grandes empresas con fines de lucro. Semejantes actores privados contribuyen, en gran medida, a la reputación internacional de las universidades y parece que sus evaluaciones están desplazando cada vez más la relevancia de las agencias nacionales entre aquellas universidades que están más atentas a los mercados globales y regionales.

Un último conjunto de críticas está relacionado con la capacidad de las agencias para promover una mejora efectiva de la calidad de las IES (Woodhouse, 2010) y sus fuentes particulares de legitimidad para introducir nuevos estándares de calidad, por ejemplo su participación en redes internacionales (Paradeise, Reale, Bleiklie, \& Ferlie, 2009). Además de esto, el número de actores involucrados actualmente en la gobernanza de las IES ha aumentado considerablemente: junto a los actores anteriormente mencionados, ahora hay autoridades locales y regionales muy involucradas; agencias independientes encargadas de evaluar las actividades universitarias; organismos de financiación centrados principalmente en apoyar la investigación; agencias de ranking que proporcionan continuamente evaluaciones comparativas; y organismos internacionales que coordinan 
operaciones a escala regional y global. La autorregulación ha disminuido, en cierta medida, y las agencias d y sus propias redes de actores se han convertido en un rasgo común en la mayoría de los sistemas universitarios en general. Por lo tanto, la gobernanza es mucho más compleja, y los instrumentos empleados son más diversos y se han vuelto mucho más sofisticados.

Por último, hay también preocupaciones acerca la fatiga de la evaluación y el aumento de la carga de trabajo que proviene de diferentes mecanismos de aseguramiento de la calidad y de otros instrumentos de rendimiento que se aplican simultáneamente (Don F. Westerheijden et al., 2007). La pérdida de la capacidad de dirección política es un tema interesante. Las agencias son bastante autónomas con respecto a los responsables políticos elegidos, pero tienen un papel importante en la formulación de políticas. Diseñan los procedimientos y el marco para la GC. La independencia política de la agencia hace que la GC sea mucho más un ejercicio técnico, que requiere principalmente conocimientos profesionales. Sin embargo, como ya se ha señalado, el ejercicio de la GC no puede considerarse independientemente de la definición del problema en la ES, que es principalmente una cuestión política. Sin embargo, esto no significa necesariamente un conflicto entre las fuerzas políticas y burocráticas. Esta GC técnica puede ser de hecho útil para ofrecer legitimidad a través procedimientos del tipo sugerido por la corriente de la Nueva Gestión Pública, nuevos procedimientos de gestión pública, incluso si no afecta al núcleo de la calidad (Enders \& Westerheijden, 2017).

\section{Conclusiones}

El análisis comparativo realizado demuestra, en primer lugar, que existen modelos de gobernanza alternativos y que son expresión de la distinta configuración de los sistemas de educación superior, tanto en términos de autonomía institucional como de de intervención estatal. Pero, en segundo lugar, el análisis también ha demostrado que existe una gran convergencia metodológica al mismo tiempo que una coincidencia en las preocupaciones que no son solo políticas, de nuevo, sino también profundamente conceptuales. De hecho, las críticas que reciben las AGC certifican, como se ha mostrado, esta convergencia que sugiere la necesidad de encontrar un nuevo equilibrio entre automía institucional, autoregulación del sector e intervención pública. Esta es la dirección hacia la que apuntan muchos gobiernos. ¿Pero será esta la dirección que aceptarán seguir las AGC? Que el gato tenga un cascabel no significa, necesariamente, que siga siempre nuestros pasos. El gato, se dice, es el animal de compañía más independiente: no acepta propietarios, pero sí el cogobierno del hogar. Tal vez el futuro conlleve, precisamente, la reformulación de las agencias como herramientas de cogobierno de la ES.

\section{Referencias}

Alzafari, K., \& Ursin, J. (2019). Implementation of quality assurance standards in European higher education: does context matter? Quality in Higher Education, 25(1), 58-75. doi:10.1080/13538322.2019.1578069

Baldwin, R., Cave, M., \& Lodge, M. (2011). Understanding Regulation: Theory, Strategy, and Practice. Oxford: Oxford University Press. 
Beerkens, M. (2016). Quality assurance in the political context: in the midst of different expectations and conflicting goals. Quality in Higher Education, 21(3), 231-250. doi:10.1080/13538322.2015.1111004

Beerkens, M. (2018). Evidence-based policy and higher education quality assurance: progress, pitfalls and promise. European Journal of Higher Education, 8(3), 272287. doi:10.1080/21568235.2018.1475248

Billing, D. (2004). International comparisons and trends in external quality assurance of higher education: Commonality or diversity? Higher Education, 47(1), 113-137. doi:10.1023/B:HIGH.0000009804.31230.5e

Blackmur, D. (2010). Does the Emperor Have the Right (or Any) Clothes? The Public Regulation of Higher Education Qualities over the Last Two Decades. Quality in Higher Education, 16(1), 67-69. doi:10.1080/13538321003679549

Brennan, J. (2018). Success factors of quality management in higher education: intended and unintended impacts. European Journal of Higher Education, 8(3), 249-257. doi:10.1080/21568235.2018.1474776

Brown, R. (2013). Mutuality Meets the Market: Analysing Changes in the Control of Quality Assurance in United Kingdom Higher Education 1992-2012. Higher Education Quarterly, 67(4), 420-437. doi:10.1111/hequ.12028

Costes, N., Curvale, B., Kraft, M. G., Llavori, R., Malan, T., \& Szanto, T. (2010). First External Evaluations of Quality Assurance Agencies--Lessons Learned. ENQA Workshop Report 10: ERIC.

Dill, D. A. (2011). Governing Quality. In R. King, S. Marginson, \& R. Naidoo (Eds.), Handbook on globalization and higher education (pp. 438). Cheltenham: Edward Elgar Publishing.

Dill, D. D., \& Beerkens, M. (Eds.). (2010). Public Policy for Academic Quality: Analyses of Innovative Policy Instruments. Berlin: Springer.

Eaton, J. S. (2011). US accreditation: Meeting the challenges of accountability and student achievement. Evaluation in Higher Education, 5(1), 1-20.

Enders, J., \& Westerheijden, D. F. (2017). The Dutch way of New Public Management. Policy and Society, 33(3), 189-198. doi:10.1016/j.polsoc.2014.07.004

Ewell, P. (2010). Twenty Years of Quality Assurance in Higher Education: What's Happened and What's Different? Quality in Higher Education, 16(2), 173-175. doi :10.1080/13538322.2010.485728

Fernández Lamarra, N. (2006). La evaluación y la acreditación de la calidad: Situación, tendencias y perspectivas. In UNESCO IESALC (Ed.), Informe sobre la educación superior en América Latina y el Caribe, 2000-2005: la metamorfosis de la educación superior. Caracas: Instituto Internacional de la UNESCO para la Educación Superior en América Latina y el Caribe. 
Green, D. (1994). What Is Quality in Higher Education? Bristol: SRHE and Open University Press.

Hopbach, A. (2012). External Quality Assurance Between European Consensus and National Agendas. In A. Curaj, P. Scott, L. Vlasceanu, \& L. Wilson (Eds.), European Higher Education at the Crossroads. Between the Bologna Process and National Reforms (pp. 267-285). Berlin: Springer.

Jarvis, D. S. (2014). Regulating higher education: Quality assurance and neo-liberal managerialism in higher education-A critical introduction. Policy and Society, 33(3), 155-166.

Jordana, J., García Juanatey, A., Pérez Durán, I., \& Sancho Royo, D. (2018). An Independence Index of Quality Assurance Agencies in Higher Education: European and Latin American countries compared. Brussels: EQAF.

Jordana, J., Levi-Faur, D., \& i Marín, X. F. (2011). The Global Diffusion of Regulatory Agencies. Comparative Political Studies, 44(10), 1343-1369. doi:10.1177/0010414011407466

Juanatey, A. G., Jordana, J., Durán, I. P., \& Royo, D. S. (2020). Independence, accountability and responsibilities of quality assurance agencies in higher education: European and Latin American countries compared. European Journal of Higher Education, 1-22. doi:10.1080/21568235.2020.1850309

Lægreid, P., \& Verhoest, K. (2010). Introduction: Reforming Public Sector Organizations. In Governance of Public Sector Organizations: Proliferation, autonomy and performance (pp. 1-18). Berlin: Springer.

Majone, G. (2008). From the Positive to the Regulatory State: Causes and Consequences of Changes in the Mode of Governance. Journal of Public Policy, 17(2), 139-167. doi:10.1017/s0143814xo0o03524

Martin, M., \& Stella, A. (2007). External Quality Assurance in Higher Education: Making Choices. Fundamentals of Educational Planning 85. Paris: UNESCO IIPE.

Olsen, J. P. (2007). The Institutional Dynamics of the European University. In University Dynamics and European Integration (pp. 25-54).

Paradeise, C., Reale, E., Bleiklie, I., \& Ferlie, E. (Eds.). (2009). University Governance. Western European Comparative Perspectives. Berlin: Springer.

Pollitt, C., Bathgate, K., Caulfield, J., Smullen, A., \& Talbot, C. (2001). Agency fever? Analysis of an international policy fashion. Journal of Comparative Policy Analysis, 3(3), 271-290.

Seyfried, M., \& Pohlenz, P. (2018). Assessing quality assurance in higher education: quality managers' perceptions of effectiveness. European Journal of Higher Education, 8(3), 258-271.

Shah, M. (2013). The effectiveness of external quality audits: a study of Australian universities. Quality in Higher Education, 19(3), 358-375. doi:10.1080/1353832 2.2013 .852300 
Siegel, D. J. (2010). Why Universities Join Cross-Sector Social Partnerships: Theory and Evidence. Journal of Higher Education Outreach and Engagement 14(1), 33-62.

Stella, A. (2006). Quality Assurance of Cross-border Higher Education. Quality in Higher Education, 12(3), 257-276. doi:10.1080/13538320601072859

Stensaker, B. (2014). European trends in quality assurance: new agendas beyond the search for convergence? In Quality Assurance in Higher Education (pp. 135-148): Springer.

Strah, M. (2020). Los sistemas nacionales de aseguramiento de la calidad de la Educación Superior en Iberoamérica. Buenos Aires: CONEAU OEI.

Talbot, C. (2004). The agency idea: sometimes old, sometimes new, sometimes borrowed, sometimes untrue. In C. Pollitt \& C. Talbot (Eds.), Unbundled government. A Critical Analysis of the Global Trend to Agencies, Quangos and Contractualisation (pp. 19-37). London: Routledge.

Thune, C.(2006). The Alliance of Accountability and Improvement: the Danish experience. Quality in Higher Education, 2(1), 21-32. doi:10.1080/1353832960020103

UNESCO IESALC. (2020). La garantía de calidad y los criterios de acreditación en la educación superior. Perspectivas internacionales. Caracas: Instituto International de la UNESCO para la Educación Superior en América Latina y el Caribe.

Verhoest, K., Peters, B. G., Bouckaert, G., \& Verschuere, B. (2004). The study of organisational autonomy: a conceptual review. Public Administration and Development, 24(2), 101-118. doi:10.1002/pad.316

Westerheijden, D. F. (2007). States and Europe and quality of higher education. In D. F. Westerheijden, B. Stensaker, \& M. J. Rosa (Eds.), Quality assurance in higher education (pp. 73-95). Berlin: Springer.

Westerheijden, D. F., Stensaker, B., \& Rosa, M. J. (Eds.). (2007). Quality Assurance In Higher Education. Berlin: Springer.

Woodhouse, D. (2003). Quality improvement through quality audit. Quality in Higher Education, 9(2), 133-139.

Woodhouse, D. (2010). The quality of quality assurance agencies. Quality in Higher Education, 1O(2), 77-87. doi:10.1080/1353832042000230572

Woodhouse, D. (2013). Global trends in quality assurance. Quality Approaches in Higher Education, 5(1), 3-7.

Yung-Chi Hou, A., Ince, M., Tsai, S., \& Chiang, C. L. (2015). Quality Assurance of quality assurance agencies from an Asian perspective. Asia Pacific Education Review, 16(1), 95-106. 